\title{
Hacia el perfeccionamiento institucional de los cuerpos académicos en la Facultad de Ingeniería Mecánica y Eléctrica de la Universidad Autónoma de Nuevo León, México
}

\author{
Towards Improving the Academic Bodies of FIME in the \\ Autonomous University of Nuevo León, México
}

\author{
María Isabel Dimas Rangel ${ }^{1}$ \\ Facultad de Ingeniería Mecánica y Eléctrica (FIME) \\ Universidad Autónoma de Nuevo León \\ Nuevo León, México \\ isabel.dimasr@gmail.com \\ Arturo Torres Bugdud ${ }^{2}$ \\ Facultad de Ingeniería Mecánica y Eléctrica (FIME) \\ Universidad Autónoma de Nuevo León \\ Nuevo León, México \\ atorres85@hotmail.com \\ Jaime Arturo Castillo Elizondo 3 \\ Facultad de Ingeniería Mecánica y Eléctrica (FIME) \\ Universidad Autónoma de Nuevo León \\ Nuevo León, México \\ jaime.castilloe@uanl.mx
}

Recibido 29 de agosto de 2012 - Corregido 22 de octubre de 2012 • Aceptado 07 de noviembre de 2012

\footnotetext{
Maestra en Ciencias de la Administración, de la Universidad Autónoma de Nuevo León (UANL). Jefa de Recursos Humanos de la Facultad de Ingeniería Mecánica y Eléctrica (FIME). Profesora titular. Miembro de la Academia de Manufactura y Calidad. Experiencia como investigadora en el área de gestión de recursos humanos y sistemas de calidad. Posee artículos publicados en las áreas de gestión de recursos humanos, gestión de procesos con base en sistemas de calidad, en revistas de prestigio internacional.

2 Ingeniero Mecánico y Eléctrico. Maestrías en Administración y en Enseñanza de las Ciencias. Doctor en Ciencias Pedagógicas. Es miembro del Sistema Nacional de Investigadores de México (SNI). Actualmente subdirector de Desarrollo Humano en FIME. Ha publicado varios artículos en revistas de prestigio internacional sobre formación de profesores y formación integral del estudiante. Lidera el cuerpo académico sobre gestión educativa. Es evaluador externo de dos revistas indexadas. Ha presentado numerosos trabajos en congresos nacionales e internacionales.

3 Ingeniero Administrador de Sistemas en la FIME de la UANL. Maestría en Administración con especialidad en Recursos Humanos. Profesor de tiempo completo con Perfil PROMEP otorgado por la Secretaría de Educación Publica de México. Experiencia en el sector empresarial y educativo para las áreas de sistemas, administración, gestión y vinculación. Actualmente subdirector de Vinculación y Relaciones de la Facultad de Ingeniería Mecánica y Eléctrica. Ha presentado y publicado diversos trabajos relacionados con el tema de la vinculación y la gestión en revistas de prestigio y congresos internacionales.
} 
URL: http://www.una.ac.cr/educare

Resumen. El presente artículo aborda un tema de crucial importancia para las instituciones de Educación Superior. En particular, en México, el trabajo colegiado de los académicos es una problemática no resuelta, a pesar de los esfuerzos que se han realizado en este sentido. Por estas razones, es imprescindible una reflexión sistemática y fundamentada sobre las potencialidades de los cuerpos académicos para el fortalecimiento de la planta docente y su consiguiente repercusión en la calidad educativa. Este artículo expone los resultados finales producto de un trabajo de investigación realizado en la FIME, que tuvo como objetivo caracterizar los cuerpos académicos en esta Facultad, de modo que se constate el estado actual y la prospectiva de los citados órganos.

Palabras claves. Cuerpos académicos, educación superior, planta académica, cuerpos colegiados, universidad, México.

Abstract. This paper refers to a crucial issue for higher education institutions. In Mexico, particularly, the collective work of academic bodies is an unresolved issue despite the efforts made in this regard. In this context, a well-founded systematic discussion is essential to understand the potential of these academic bodies on faculty strengthening and their subsequent impact on the quality of education. This paper presents the results of a research project conducted by $\mathrm{FIME}^{4}$ with the purpose of identifying the characteristics of its academic bodies as well as their current and potential condition.

Keywords. Academic bodies, Higher Education, academic staff, collegiate bodies, University, México.

\section{Antecedentes y justificación del estudio}

Es reconocido que las instituciones de educación superior (IES) juegan un papel muy importante en el desarrollo de un país, por constituir la principal fuente de potencialidad humana, a la par que propician una transformación necesaria para el desarrollo de la sociedad. Además, promueven la generación de conocimientos científicos y tecnológicos que influyen en el desarrollo y consolidación de los diferentes órdenes de la vida sociopolítica del país. Para contribuir positivamente con estos retos, es necesario que la universidad apoye y propicie el desarrollo de su planta académica, por ser esta una clave importante para el cumplimiento de los objetivos institucionales, para el fortalecimiento de los programas educativos $y$, por ende, de la elevación de la calidad de los egresados.

En este sentido, el Programa de Mejoramiento del Profesorado (PROMEP), creado en 1996, muestra que los profesores de carrera y su habilitación, dedicación y desempeño en los cuerpos académicos de las universidades públicas de México constituyen un medio estratégico para elevar la calidad de la educación superior (PROMEP, 2006). Por otra parte, en 2005, a través de los Programas Integrales de Fortalecimiento Institucional (PIFI), la Secretaría de Educación Pública (SEP) invirtió, en el citado programa de mejoramiento, 390.3 millones de pesos para mejorar el perfil académico y la habilitación del profesorado, así como el desarrollo, fortalecimiento y consolidación de los cuerpos académicos (PROMEP, 2006).

4 Translator's Note: FIME refers to the Facultad de Ingeniería Mecánica y Eléctrica (College of Mechanical and Electrical Engineering). 
Es reconocido que la relación de las profesiones con la sociedad y el conocimiento válido puede concebir la profesión académica "(...) como forma legítima e integrada de agrupación ocupacional. Representa la asociación de actores que se ubican en las organizaciones de educación superior para cumplir con un fin, la función de producir, transmitir y certificar la adquisición del conocimiento (...) (Grediaga, 2000, pp. 160-161). Para este autor, los académicos constituyen un tipo específico de rol ocupacional, por ser los responsables directos del proceso de enseñanza-aprendizaje, que concluye con la obtención de grados (certificados) otorgados por las instituciones; porque realizan tareas de investigación para desarrollar el conocimiento en los distintos campos del saber especializado, y porque, a partir del desarrollo de estas actividades, influyen y favorecen el proceso de preparación requerido por las distintas profesiones.

Los argumentos expresados indican el reconocimiento de la función social de las universidades. Sin embargo, estas instituciones han de trazar estrategias concretas que permitan el crecimiento personal y profesional de sus profesores; una de las vías para ello pueden ser las estructuras ya existentes, pero con una transformación en su concepción y desarrollo. Las estrategias de mejora pueden ser concretadas mediante los cuerpos académicos (CA), siempre y cuando estén integrados por profesores y profesoras con un alto compromiso con la educación en beneficio de la sociedad, mediante la búsqueda de la excelencia en las actividades de docencia e investigación, así como de la gestión universitaria.

\section{Metodología}

El presente estudio tiene una naturaleza de carácter exploratorio, con interés en un fenómeno específico: los cuerpos académicos. Estos se analizan desde una perspectiva cualitativa conjuntamente con la utilización de técnicas de recolección de datos. Se usa este enfoque para analizar el significado de los objetivos y funciones de los cuerpos académicos a partir del propio marco de referencia del contexto objeto de estudio.

Se usaron múltiples fuentes de información que permitieron obtener una perspectiva actual sobre el tema abordado. Se recogió la información mediante entrevistas y cuestionarios aplicados a líderes y miembros de los CA. Estas técnicas permitieron conocer aspectos medulares relacionados con el desarrollo e impacto de los CA en la FIME.

El cuestionario estuvo integrado por diferentes tipos de preguntas que permitieron, por una parte, centrar las respuestas en los aspectos fundamentales y; por otra, recibir de los sujetos encuestados, aportaciones para completar la comprensión de los elementos explorados. Los datos fueron analizados a partir de la identificación de códigos temáticos, que permitieron la conceptualización, categorización, organización y estructuración en tablas y figuras. Además, se sintetizaron los criterios fundamentales emitidos en las preguntas abiertas. 
URL: http://www.una.ac.cr/educare

La investigación se sitúa dentro del campo de estudios de políticas públicas con delimitación en el proceso de implementación, esto es, en el estudio de las condiciones con las cuales las decisiones de la autoridad conducen o no, efectivamente, al logro de los resultados establecidos (Berman, 2000).

\section{Muestra}

Se aplicó el instrumento diseñado a los 23 líderes de CA en FIME. De ellos contestaron la encuesta el 69\%. Asimismo, dicho instrumento fue aplicado a 94 docentes integrados a diferentes cuerpos académicos, lo que representa el $36 \%$ del total.

\section{Desarrollo}

\section{Actualidad, perspectivas e importancia de los cuerpos académicos}

Es evidente que, con el desarrollo de la ciencia y la tecnología, se acercan las fronteras entre las diferentes ciencias y entre temas de una misma ciencia. Por ello, el trabajo colectivo se ha convertido en una consecuencia lógica de este desarrollo y, a su vez, una necesidad insoslayable para la obtención de productos también acordes con las nuevas exigencias.

El trabajo individual, en cualquier rama del saber, no puede abarcar las diferentes aristas del estudio de un determinado fenómeno o proceso; por tanto, la organización de las actividades en las universidades con un enfoque colegiado, más que una disposición administrativa, constituye una necesidad impostergable. De ahí que en diferentes países en las instituciones de educación superior, existan órganos científicos y metodológicos que, de una u otra forma, integran a los docentes, con vistas a la realización de trabajos y proyectos colectivos -ya sean de carácter investigativo o de otra índole-. Los más comunes se relacionan con colectivos de investigadores que despliegan de manera colegiada investigaciones aplicadas o generadoras de conocimiento en las diferentes ramas del saber.

La necesidad de colaboración entre académicos ha sido ampliamente reconocida. En México, la ANUIES (Asociación Nacional de Universidades e Instituciones de Educación Superior), destaca que:

(...) La mayor interacción entre las comunidades académicas permite un proceso continuo de mejoramiento de la calidad educativa; la apertura a la interacción mundial potencia los procesos de transformación de las instituciones educativas, y el surgimiento de nuevos valores en la sociedad permite la construcción de espacios académicos más consolidados (...). (ANUIES, 2000, p. 15) 
En ocasiones, cuando se alude al trabajo en colectivo, se aplica el término "comunidades epistémicas", el que se refiere a grupos de expertos que tienen como misión resolver una serie de problemas a través de la aplicación del conocimiento científico (Maldonado-Maldonado, 2005). Por otra parte, Kuhn, citado por Olivé (2008), expresa:

(...) casi todos los científicos asumen una filiación comunitaria, y dan por supuesto que la responsabilidad de llevar a cabo las tareas que corresponden a las diferentes especialidades de la disciplina se distribuyen entre grupos cuya membrecía está más o menos determinada (...). (p. 30)

En otros casos, se hace referencia a "comunidades científicas, cuyo objetivo es generar un auténtico conocimiento en su campo, un conocimiento objetivo de la realidad que sea resultado de procesos racionales (...)". Se agrega que "(...) las comunidades científicas se caracterizan por una constelación de elementos compartidos; entre ellos, los conocimientos previos que acumularon en su campo, pero sobre todo, un conjunto de valores e intereses comunes dentro de cada especialidad (...) (Olivé, 2008, p. 33).

Como se destaca, uno de los requisitos para el establecimiento de la actividad colaborativa recalcado por Tierney (2001) como su elemento principal, es la producción de conocimiento mediante el trabajo conjunto. Advierte que la "comunidad de científicos dentro de un campo específico trabaja conjuntamente, de modo que una persona aprende un hecho y otra construye sobre este hecho para descubrir otro, y así sucesivamente (...)" (p. 165).

A partir de lo antes expuesto, el cuerpo académico constituye una pequeña comunidad científica que produce y aplica conocimiento mediante el desarrollo de una o varias líneas de investigación. A pesar de que, de acuerdo con el desarrollo actual, este tipo de comunidad u organización posee importancia indiscutible, no siempre los docentes están conscientes de ello, por lo que es imprescindible que este enfoque de colaboración sea asumido, por la planta académica, como una respuesta a las necesidades sociales, profesionales y personales.

Se comparte con Acosta (2006, pp. 89-90) la idea de que "(...) querer creer que la formación de cuerpos académicos permitirá a las universidades, ahora sí, contar con académicos ordenados, cooperativos y productivos, bien calificados, con objetivos precisos y compromisos claros, es la muestra de un optimismo desmesurado, un tanto fantasioso, por los efectos que pueden tener los programas respecto de la situación que prima en los ambientes académicos universitarios (...)". En este sentido, "(...) no hay que olvidar que la preparación del personal académico y su agrupación en cuerpos académicos constituyen los pilares fundamentales donde descansa la mejora de la calidad de la educación (...)" (ANUIES, 2000, p. 104).

Significa que los docentes han de estar conscientes de la importancia que cada día tienen los cuerpos académicos porque, como expresa Fresán (s. f.), es obvio que: 
URL: http://www.una.ac.cr/educare

Los cuerpos académicos no son agregaciones artificiales de académicos integradas por profesores:

- que no han trabajado juntos

- que tienen poca experiencia en investigación

- que no comparten objetivos académicos

- que no comparten objetos de estudio. (p. 14)

\section{Conceptualización, objetivos y caracterización de los cuerpos académicos}

El concepto sobre cuerpo académico más generalizado es el aportado por PROMEP, citado por López (2010), donde se define como "(...) conjunto de profesores-investigadores que comparten una o más líneas afines de investigación (estudio), cuyos objetivos y metas están destinados a la generación y/o [sic] aplicación de nuevos conocimientos, además de que, a través de su alto grado de especialización, los miembros ejerzan la docencia para lograr una educación de buena calidad" (p. 8).

Esta definición se enriquece por algunos autores (Martínez, Rico y Preciado, 2006):

(...) Los cuerpos académicos se sustentan en el proceso formativo de los estudiantes a través de y para [sic] el quehacer científico, de ahí que, aunque existan condiciones de infraestructura idóneas, buenos programas, alumnos brillantes y con un potencial de aprendizaje demostrado, la carencia de una planta académica organizada alrededor de líneas y proyectos de investigación impide que se dé el ambiente y la organización académica necesarias [sic] para el éxito de los programas en los diferentes niveles de formación. (pp. 21, 23)

Sin embargo, la realidad demuestra que el reconocimiento de la importancia y necesidad de este tipo de trabajo no es suficiente para lograr los propósitos que justifican su surgimiento. Se está de acuerdo con Acosta $(2006$, p. 86) quien expone que:

(...) se parte de la creencia de que los académicos, independientemente de las disciplinas, contextos institucionales, tradiciones, creencias y hábitos de trabajo, tienden a agruparse para producir conocimiento, trasmitirlo o difundirlo. $Y$ aquí, como ocurre con todas las creencias, hay problemas. No todos los académicos en todas las disciplinas actúan así, ni todo el tiempo (...). 
Diferentes estudios han puesto de manifiesto que, a medida que transcurre el tiempo, la planta académica va concientizando la importancia de este tipo de órgano. Según Guzmán, Hernández y Guzmán (2009), el cuerpo académico es visto por el profesor "como un ente que genera liderazgo académico en la unidad académica, escuela o facultad a la cual pertenece, da impulso institucional para la formación de cuadros académicos orientados a promover la investigación y como un medio para integrarse a redes temáticas y de colaboración con instituciones nacionales e internacionales" (p. 65).

Empero, no se puede aseverar que en la actualidad los cuerpos académicos muestren los resultados deseados. López (2010) corrobora esta idea en un estudio realizado, donde se muestran resultados de 275 profesores. El citado autor expone que:

(...) se insistió en que existen dos elementos que limitan el fortalecimiento de los cuerpos académicos: el primero es el hecho de que los miembros produzcan prácticamente de manera individual, y el segundo es que la formación de un cuerpo académico sea puramente organizacional, impuesta desde la SEP. (p. 17)

Este segundo aspecto se relaciona directamente con el tema de la motivación para integrar un cuerpo académico, aspecto determinante para el establecimiento de un clima adecuado de colaboración.

De los 275 profesores encuestados, 158 coincidieron en que los elementos de mayor peso en el fortalecimiento de su cuerpo académico están relacionados con la producción y aplicación del conocimiento, acaparando [sic] el 57.5\% de las respuestas obtenidas; mientras que 81 investigadores, 29.5\%, afirmaron que es la definición de una agenda común e intereses compartidos la de mayor peso, posicionando [sic] a este elemento en segundo lugar; y por último, se consideró a los elementos administrativos y de organización, con un 7.2\%. (López, 2010, p. 17)

De acuerdo con los datos recabados, otros de los elementos que motivan la producción de conocimiento son: el trabajo colectivo, la eficiencia de las políticas de financiamiento, la existencia de perspectivas teórico-metodológicas similares entre los miembros, condiciones de trabajo y la consolidación de las líneas de investigación. Por otro lado, un elemento que impide el fortalecimiento de la producción de conocimiento es la burocracia. (López, 2010, p. 18) 
URL: http://www.una.ac.cr/educare

Como se observa los CA poseen muchas potencialidades; sin embargo, no todas son reconocidas por el profesorado, según expresa (López, 2010). No obstante, el citado autor hace referencia a tres elementos que gozan de una mayor aceptación, según un estudio realizado, de los cuales el de mayor importancia es la producción de conocimiento, con 160 opiniones a favor que representa el 58.2\%; en segundo lugar, la solución de problemas de la sociedad con 59 respuestas, equivale al $21.5 \%$, y, en último lugar, la búsqueda de prestigio y credenciales académicas con 38 opiniones que representan el 13.8\% de los profesores encuestados.

Otros elementos que procuran los miembros de los cuerpos académicos, según los profesores objetos del estudio mencionado, son: la formación de recursos humanos de alto nivel (licenciatura, maestría y doctorado); una mayor eficiencia académica; un mayor impacto académico mediante el establecimiento de formas de trabajo colectivo, interdisciplinario e interinstitucional, y la consolidación de redes con otros cuerpos académicos. Algunos profesores señalan que hay quienes solo buscan la obtención de un buen financiamiento, para intereses particulares. Estos datos son indicadores importantes acerca del significado y sentido que poseen los cuerpos académicos para los profesores universitarios y han servido de referentes para el presente estudio.

\section{Desarrollo de los cuerpos académicos}

Independientemente de que hasta la fecha no existen procedimientos claros para la evaluación de los cuerpos académicos, tal y como plantea Fresán (s. f.) cuando expresa que una preocupación compartida por los académicos mexicanos es la necesidad de "hacer públicos los criterios de evaluación de los cuerpos académicos y los estándares que permiten ubicarlos dentro de las distintas categorías" (p. 15), existen algunos indicadores que permiten valorar su solidez. Varios autores han hecho referencia a las características de cada etapa del desarrollo del CA (Martínez et al., 2006; Suárez y López, 2006), tomando como punto de partida los rasgos que se plantean en el Programa de Mejoramiento del Profesorado (Promep) (2006).

En los trabajos donde se aborda lo relacionado con el desarrollo de los cuerpos académicos se concuerda en la precisión de determinadas características para cada etapa de desarrollo. Estos aspectos coincidentes se relacionan a continuación. Esta caracterización cobra una importancia metodológica ya que constituye una orientación importante para la evolución de estos órganos.

\section{Cuerpo académico en formación:}

- Poseen la identificación de sus integrantes.

- Una minoría significativa de sus miembros presenta el perfil deseable con el grado preferente o, por lo menos la mitad, con grado mínimo. 
- Tienen bien definidas las líneas de generación y aplicación del conocimiento.

- Ostentan contactos con cuerpos académicos afines y de alto nivel, de otras instituciones del país o del extranjero con quienes desean establecer vínculos de colaboración.

- Están informados de la plataforma básica que necesitan para su trabajo.

\section{Cuerpo académico en consolidación:}

- La mitad de sus integrantes posee el grado preferente y cuenta con productos sólidos de generación o aplicación del conocimiento.

- Una mayoría tiene reconocimiento del perfil deseable.

- Participan conjuntamente en líneas de generación o aplicación del conocimiento.

- La mayoría de sus miembros participa activamente en redes de intercambio con sus pares en el país y en el extranjero.

- Por lo menos la tercera parte cuenta con amplia experiencia en docencia y en formación de recursos humanos.

- Poseen parte de la plataforma básica necesaria para su trabajo y tienen identificados sus requerimientos para completarla.

\section{Cuerpo académico consolidado:}

- La mayoría de sus integrantes tienen la misma habilitación académica que los capacita para generar y aplicar innovadoramente el conocimiento de manera independiente.

- Cuentan con amplia experiencia en docencia y en formación de recursos humanos.

- Mantienen un alto compromiso institucional que se manifiesta en una relación con la institución más allá de la contractual y una amplia participación en docencia y en las demás tareas universitarias.

- Sostienen una intensa participación en redes de intercambio académico, con sus pares en el país y en el extranjero, así como con organismos e instituciones nacionales y del extranjero.

\section{Hacia el perfeccionamiento de los cuerpos académicos en la FIME}

Los argumentos analizados constituyen claves importantes para que la Universidad Autónoma de Nuevo León (UANL), tercera universidad de México, así como la institución pública de educación superior más importante del noreste del país (UANL, 2011), le otorgue 
URL: http://www.una.ac.cr/educare

una importancia de primer orden al desarrollo de sus cuerpos académicos. Desde hace varios años esta institución se ha orientado a implementar estrategias que alcancen estándares de calidad que puedan ser reconocidos por organismos acreditadores y certificadores nacionales e internacionales.

Por su parte, la Facultad de Ingeniería Mecánica y Eléctrica, de la UANL, no está al margen de los objetivos antes mencionados: por ser la más grande de la Institución, el compromiso también es mayor. Cuenta con 10 carreras a nivel de Licenciatura, 14 programas de Posgrado y más de 100 cursos de Educación Continua (FIME, 2011). Son altos los retos para lograr las expectativas de estudiantes, organismos empleadores y la sociedad en general, al estar obligados a formar profesionales competitivos, reconocidos por la excelencia de su trabajo, formados integralmente y con una visión clara de su compromiso social. Esta visión clasifica su misión en el logro de determinados objetivos de calidad, orientados a abarcar aspectos importantes en la consolidación de los resultados a obtener por la facultad. Dichos objetivos se relacionan con:

1.- Contar con una amplia y diversificada oferta educativa en ingeniería, reconocida por su calidad, para la formación de profesionales y científicos, competentes a nivel nacional e internacional y con una alta adaptabilidad en el mundo laboral.

2.- Ser un polo de desarrollo científico, tecnológico, académico, humanístico, de alto impacto social, por sus contribuciones relevantes a la generación y aplicación del conocimiento, así como en la atención a problemáticas significativas del desarrollo social y económico del Estado y del país.

3.- Contribuir a la formación integral de los estudiantes a través de la participación en actividades relacionadas con la gestión y la divulgación del conocimiento donde se logre una alta participación en eventos sobre las diferentes áreas de conocimiento.

4.- Implementar una gestión eficiente y eficaz, de apoyo al desarrollo de las funciones educativas y científicas, con esquemas para la mejora continua, el aseguramiento de calidad y para el ejercicio transparente, con la rendición de cuentas a la sociedad.

5.- Poseer un alto nivel de posicionamiento y reconocimiento social en los ámbitos nacional e internacional. (FIME, 2011, Objetivos de calidad, párr. 1)

\section{Visión diagnóstica y prospectiva de los cuerpos académicos en FIME}

La experiencia de los autores, la revisión de las fuentes teóricas, el análisis documental sobre los cuerpos académicos, así como los resultados del cuestionario aplicado, permiten una aproximación a la determinación de claves importantes sobre los cuerpos académicos tanto en su "ser" como en su "deber ser". 


\section{Importancia de los cuerpos académicos}

Resultan interesantes los elementos aportados por líderes y miembros de los CA sobre la importancia de los cuerpos académicos. La mayoría de los encuestados le atribuyen importancia a los cuerpos académicos y precisan que las actividades que se desarrollan con mayor sistematicidad son producción y aplicación del conocimiento, planeación de actividades a desarrollar tanto colectivas como individuales y acciones vinculadas a los programas educativos (Ver figura 1).

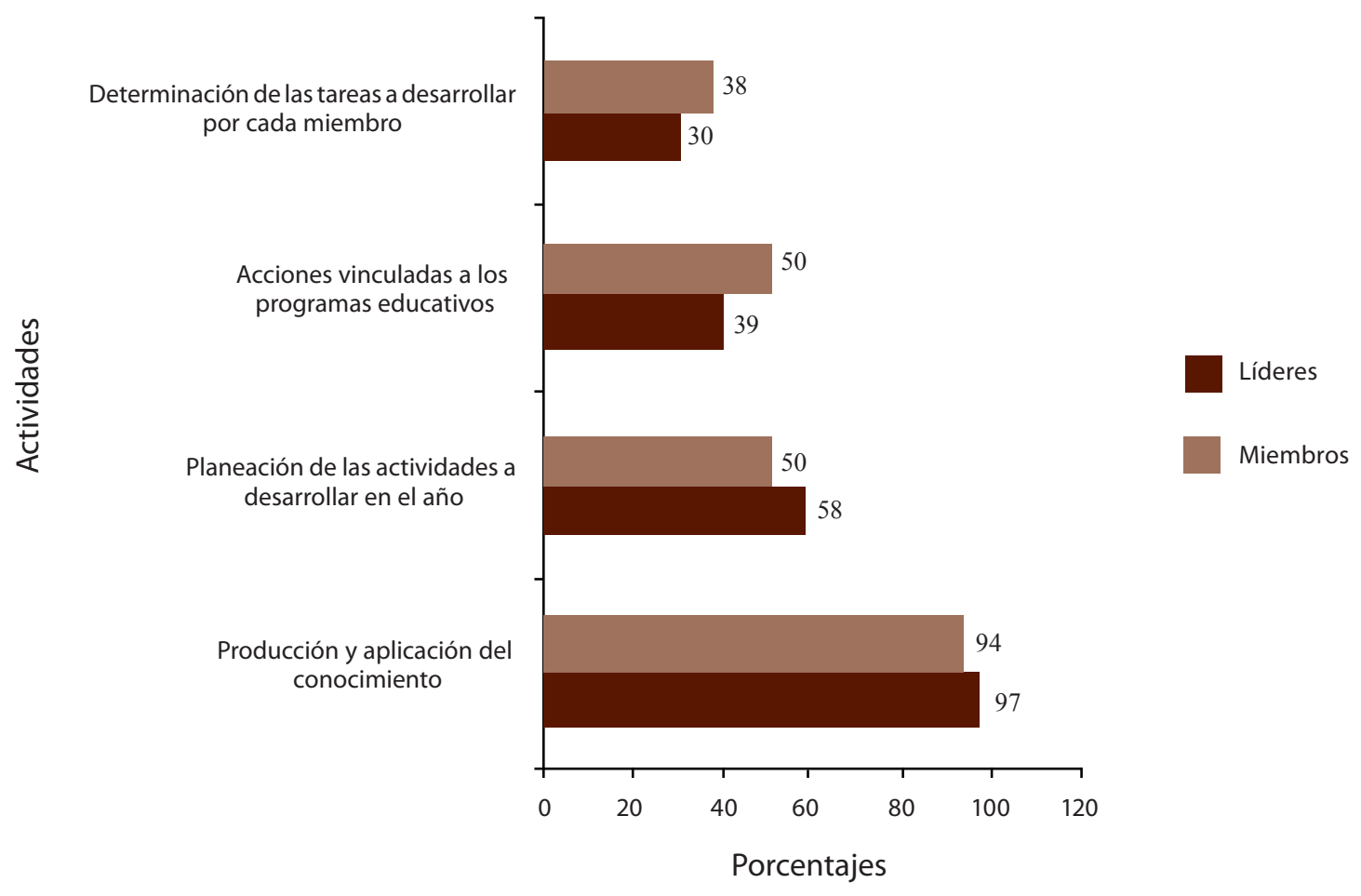

Figura 1. Actividades que se desarrollan de manera sistemática por los CA

La mayoría de los líderes y los docentes reconocen la importancia de los CA, entre los argumentos expuestos a través de la entrevista se encuentran los siguientes:

Criterios de quienes reconocen la importancia de los cuerpos académicos

Los criterios más frecuentes de quienes reconocen la importancia de los CA se pueden agrupar en las siguientes categorías:

1. Fortalecimiento del trabajo en equipo

2. Desarrollo colectivo e individual de los miembros 
URL: http://www.una.ac.cr/educare

3. Desarrollo de líneas de investigación y proyectos

4. Desarrollo del conocimiento de frontera

5. Obtención de recursos para aplicación de conocimientos

6. Transferencia tecnológica y formación de recursos humanos

7. Medio empleado para fortalecer las academias

8. Producción científica y divulgación de resultados

Como se observa, los criterios destacan las ventajas de los CA para la colaboración, la comunicación entre los docentes, su influencia en el fortalecimiento de la planta académica y también su impacto en las academias. Es de destacar que el mayor énfasis se orienta hacia la realización de proyectos de investigación y, en menor medida, se ha concientizado la posible influencia de estos órganos en el proceso formativo del estudiante.

Criterios de quienes no reconocen la importancia de los CA

Aunque en pocos casos, algunos de los miembros de los CA no reconocen el valor de este tipo de organización.

- Poca relevancia: no hay suficientes profesores en líneas específicas de conocimiento

- Carencia de reconocimiento del trabajo de estos cuerpos

- Insuficiente financiamiento

- Poco apoyo; cuando existe es para los nuevos CA

- Objetivos desconocidos: Lo más grave es que no se conoce cuáles son los objetivos para los cuales fueron creados

Criterios de quienes reconocen la importancia pero bajo determinadas condiciones

Existe un tercer grupo que incluye a los líderes y miembros que reconocen el significado de los CA, pero realizan observaciones a su funcionamiento.

- Técnicamente, serían un grupo importante de apoyo en la realización de trabajo científico-académico. Sin embargo, no se poseen buenos espacios para realizar este tipo de actividades. 
- Los maestros de licenciatura no cuentan con horas dedicadas a la investigación, lo que complica la realización de las actividades requeridas para un buen funcionamiento de un CA.

- Se ocupan salas de juntas para que se reúnan los CA que estén equipados con pizarrones, acceso a internet, un proyector, etc. sin estar peleando por el espacio con organismos administrativos.

- Un CA no únicamente ocupa una sala para una breve reunión organizacional, sino también para sesiones de trabajo colaborativo, ya que las oficinas son chiquitas y algunos ni siquiera cuentan con una oficina propia.

\section{Contribución de los cuerpos académicos al desarrollo de la planta docente}

Las respuestas sobre la contribución que pueden hacer los CA al desarrollo de la planta docente, como a sus objetivos fundamentales, aportadas en la entrevista y una de las preguntas abiertas del cuestionario, se clasificaron también en tres grupos: Los que consideran que sí influyen el desarrollo de la planta académica; el que considera que influyen solo si se cumplen determinados requisitos y un tercer grupo que expresa que no ofrecen contribución alguna.

Criterios de quienes reconocen el aporte de los cuerpos académicos al desarrollo de la planta académica

$\checkmark$ Permiten compartir objetivos comunes: Mediante el trabajo en equipo se pueden lograr metas personales y del grupo en un tiempo más corto, además de que cumplir con los indicadores en cada revisión del CA es una oportunidad de mejorar.

$\checkmark$ Permiten el desarrollo de los profesores mediante su intervención en los proyectos de investigación o desarrollo y evidencian su impacto en la sociedad, con la divulgación de sus resultados.

$\checkmark$ Mantienen activo al participante al pertenecer a un CA o trabajar en un proyecto de investigación. Además, contribuyen a la creación de artículos científicos y especialización de la planta docente.

$\checkmark$ Crean vínculos con otros cuerpos colegiados para intercambiar experiencia profesional.

$\checkmark$ Inducen a los profesores a una dinámica de superación y actualización continua.

$\checkmark$ Promueven en los maestros el interés de pertenecer a ellos con el afán del estatus académico. 
URL: http://www.una.ac.cr/educare

Criterios de quienes reconocen el aporte de los cuerpos académicos al desarrollo de la planta académica solo si existen determinados requisitos

$\checkmark$ Pudieran contribuir al desarrollo de los profesores, si realmente existe un apoyo de los departamentos dentro de los cuales se encuentra el cuerpo, es cierto que se contribuye realmente a la mejora.

$\checkmark$ Aportan más a la investigación que a la docencia.

$\checkmark$ Siempre y cuando haya colaboración en el trabajo, ya que de esta manera los productos académicos son compartidos y de beneficio para todos.

$\checkmark$ Siempre que haya aportes por parte de todos, es común que en los cuerpos se infiltren compañeros que no aportan.

$\checkmark$ Los cuerpos académicos tienen la oportunidad de contribuir al desarrollo de la planta docente siempre que se les dé la oportunidad de compartir sus temas de investigación y sus diferentes resultados, con el resto de los docentes de las diferentes facultades.

$\checkmark$ Cuando operan bien, sí. De cierta forma es como un programa de mentoreo/inducción grupal, para las personas que ingresan al plantel docente.

Criterios de quienes no reconocen el aporte de los cuerpos académicos al desarrollo de la planta académica

$\checkmark$ No aportan, nadie los conoce, no se sabe qué hacen.

$\checkmark$ Hay CA que solo existen para fines de cumplir con los requisitos de los estímulos.

$\checkmark$ Algunos docentes no están en ningún cuerpo, no tienen perfil PROMEP, lo que hace que la planta docente se mantenga ineficiente y no desarrolle las expectativas ni las tendencias que se requieren en el contexto del plan de desarrollo.

Tanto los líderes como los profesores-miembros, realizaron recomendaciones en el sentido apuntado. Al respecto, expresaron lo siguiente:

$\checkmark$ Falta de facilidades para los maestros de licenciatura en participar en estas actividades. Cuestión que debe fortalecerse.

$\checkmark$ Se podría mejorar la contribución de los CA al desarrollo docente, a través de coloquios semestrales, donde los diferentes cuerpos académicos de las facultades presentaran, de manera concreta, los resultados de sus investigaciones. 


\section{Impacto de los cuerpos académicos en el perfeccionamiento de los programas educativos}

Uno de los aspectos esenciales en el trabajo de los cuerpos académicos es el relacionado con el impacto de los CA en el perfeccionamiento de los programas educativos, por ser el proceso formativo de los estudiantes, razón esencial de las universidades. En este sentido, aunque el mayor porcentaje (44\%) de respuestas positivas corresponde a los líderes, también la mayoría de ellos (12\%), expresaron que no impactan. Al sumar los porcentajes de la categoría "si y "en parte" se obtiene un $88 \%$ por parte de los líderes y un $94 \%$ por parte de los docentes (Ver figura 2).

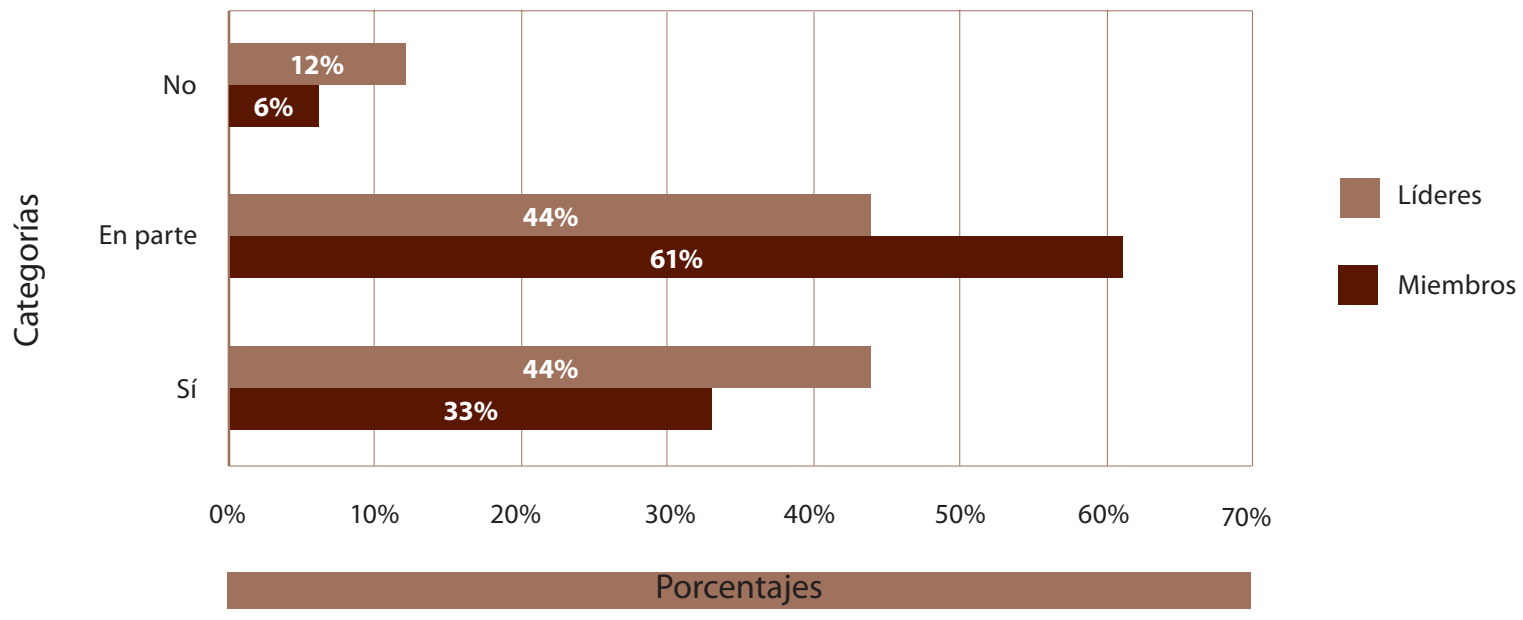

Figura 2. Impacto de los CA en los programas educativos

Criterios de los que consideran que los cuerpos académicos impactan en los programas educativos

- Al desarrollar las líneas de generación y aplicación del conocimiento (LGAC) se involucra a profesores y estudiantes, con diversas actividades que permitan mantener actualizados los currículos y el desarrollo de las competencias.

- Los cuerpos académicos, al trabajar en las fronteras del conocimiento de sus respectivas áreas, tienen la oportunidad de brindar perspectivas novedosas.

- Uno de los principales impactos de los cuerpos académicos sobre los programas educativos es el de enriquecerlos y complementarlos.

- El CA facilita la publicación de artículos en co-autoría con los miembros de CA. 
URL: http://www.una.ac.cr/educare

- Dirección y codirección de tesis.

- Mediante proyectos avanzados relacionados con el desarrollo de última generación que mantienen al programa educativo actualizado.

- Permite incorporar nuevas tecnologías y conocimiento en los estudiantes, además de incorporarlos en trabajos de investigación y fomentar sus estudios de posgrado.

- Constituyen el elemento central para hacer coincidir el trabajo colegiado de profesores y alumnos para el desarrollo de las líneas de generación y aplicación del conocimiento que permiten la formación académica y humanista e integral del estudiante universitario.

Criterios de los que consideran que los cuerpos académicos no impactan en los programas educativos

- Las recomendaciones que emanan de los CA no se toman en cuenta.

- La participación de un CA se ha convertido en un formalismo requerido y en realidad no aportan mucho.

- La reorganización de academias se realizan más bien por "política" y razones administrativos, sin considerar las recomendaciones realizadas por un CA en el área.

Criterios de los que consideran que los cuerpos académicos podrían impactar si se tomaran en cuenta sus recomendaciones:

- En la elaboración de los programas analíticos y la asignación de este trabajo al profesorado, el consultar con un CA podría ser muy valioso, pero no se realiza en mi área.

- Frecuentemente los jefes de carrera realizan las actividades, al parecer, según su juicio individual, sin tomar en cuenta el cuerpo académico.

- El impacto debería ser directo; sin embargo, actualmente las actividades de los CA no se cruzan en la planeación de los programas educativos, sino que el impacto es indirecto, se informa que hay mejoras, pero los resultados no son medibles.

- El impacto depende del grado de compromiso de los integrantes del cuerpo.

- Cuando se les toma en cuentas es trascendental, pero generalmente no ocurre de esta manera.

- Los profesores de un cuerpo académico pueden incrementar el nivel de los programas educativos, pero son ellos en lo personal, no por pertenecer a este órgano.

- Existen pruebas de que hay profesores que aportan a la parte académica mucho antes que existieran cuerpos académicos. 
A través de la entrevista y el cuestionario se ofrecieron ejemplos de cómo el cuerpo académico al que pertenecen influye en el mejoramiento de los programas educativos. A continuación se relacionan los ejemplos más frecuentes, los que se han agrupado en varias categorías:

1. Obtención de recursos.

2. Inclusión de estudiantes en proyectos de investigación o desarrollo para la vinculación con problemas reales de su profesión.

3. Contribución a la frontera de conocimiento en los campos de competencia del CA asociados a diversos programas.

4. Dirección de tesis de maestría y doctorado.

5. Vinculación con otras instituciones de educación superior (nacionales e internacionales).

6. Actualización de los profesores.

7. Perfeccionamiento del diseño curricular.

8. Elaboración de textos.

9. Publicaciones conjuntas (profesores-estudiantes).

10. Preparación de cursos de capacitación.

Los ejemplos mencionados por los encuestados muestran que es reconocido el impacto que los CA pueden tener en los diferentes programas educativos de la Facultad, no obstante se nota cierto vacío en cuanto al vínculo necesario docencia-investigación.

\section{Prospectivas de los cuerpos académicos}

Relacionado con lo anterior, tanto líderes como miembros enunciaron los aspectos que, desde su perspectiva, permiten un buen desempeño de los cuerpos académicos (Ver tabla 1). Según los datos los más influyentes en el sentido apuntado son: saber trabajar en equipo, tener claros los objetivos y metas a alcanzar, así como las buenas relaciones entre sus miembros. Estos resultados coinciden en gran medida con los obtenidos por Acosta (2006) y López (2010). 
URL: http://www.una.ac.cr/educare

Tabla 1

Factores de mayor influencia en el desempeño de los cuerpos académicos

\begin{tabular}{lcc}
\hline Factores & Miembros & Líderes \\
\hline Saber trabajar en equipo & $88 \%$ & $94 \%$ \\
Tener claros los objetivos y metas a alcanzar & $82 \%$ & $94 \%$ \\
Estar comprometido con el desarrollo de las IES & $52 \%$ & $44 \%$ \\
Los deseos de contribuir al desarrollo social & $12 \%$ & $0 \%$ \\
Actuaron con valores éticos & $18 \%$ & $6 \%$ \\
Las buenas relaciones entre sus miembros & $55 \%$ & $50 \%$ \\
Otros & $3 \%$ & $13 \%$ \\
\hline
\end{tabular}

De acuerdo con los objetivos de la investigación, también resultaba de interés constatar la opinión de líderes y miembros sobre los valores que posibilitan el buen trabajo de los CA. Se observa en la figura 3, los valores que se consideran más importantes son: la colaboración (94\% respectivamente) y la disciplina (88\% y 94\%). Resulta de interés para el trabajo futuro de los CA la constatación acerca de que la humildad y la colaboración obtuvieron valores relativamente bajos. Es conocido que estos dos valores guardan una estrecha relación y son imprescindibles para el logro de un clima favorable que propicie los mejores resultados del trabajo en común.

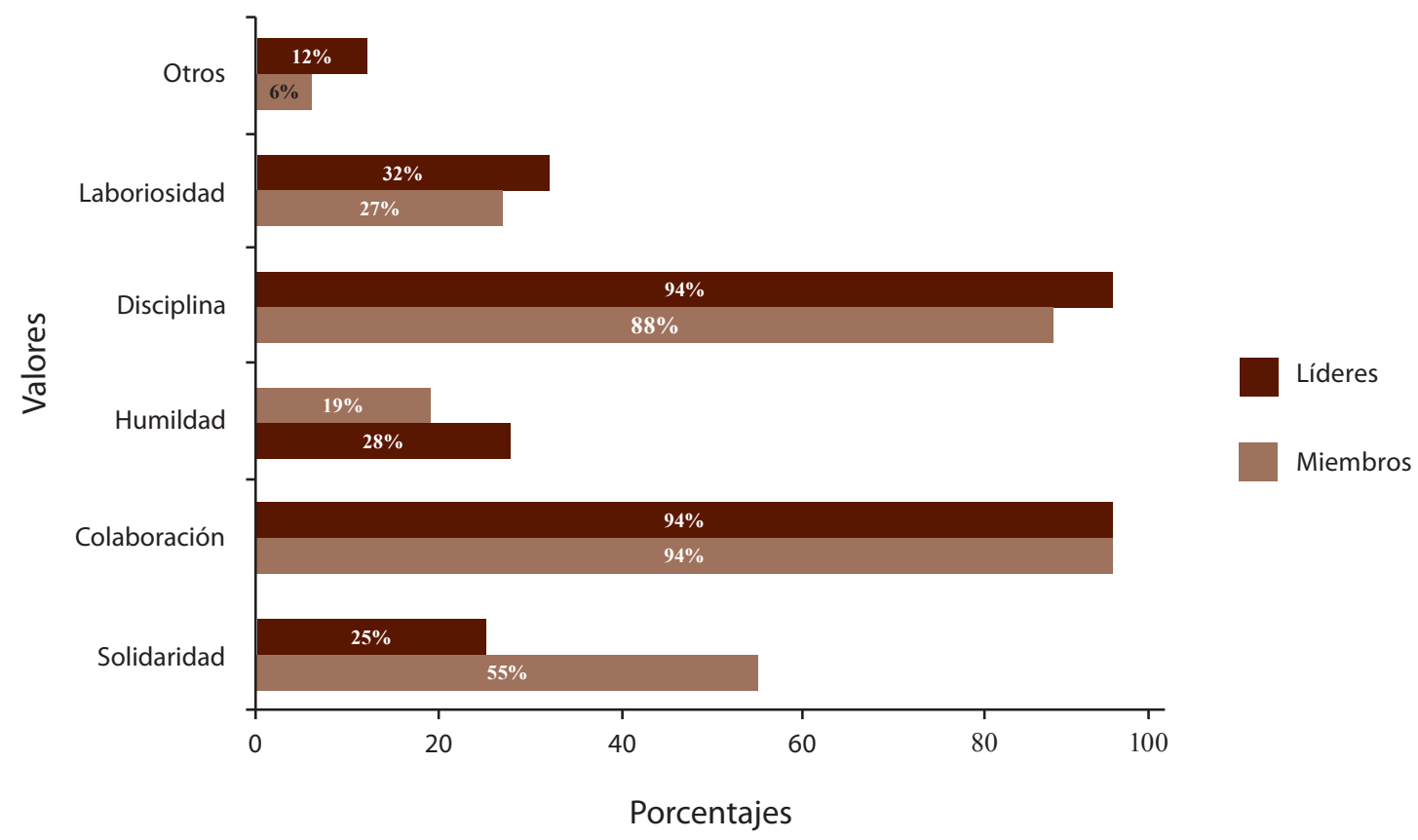

Figura 3. Valores que influyen en el buen trabajo de los cuerpos académicos 
Entre los factores que más afectan el buen desempeño de los cuerpos académicos se encuentran: la falta de comunicación entre los miembros y la falta de organización del trabajo; en menos medida, pero con valores nada despreciables aparecen: el individualismo y las limitaciones en el desarrollo individual de los miembros (Ver figura 4).

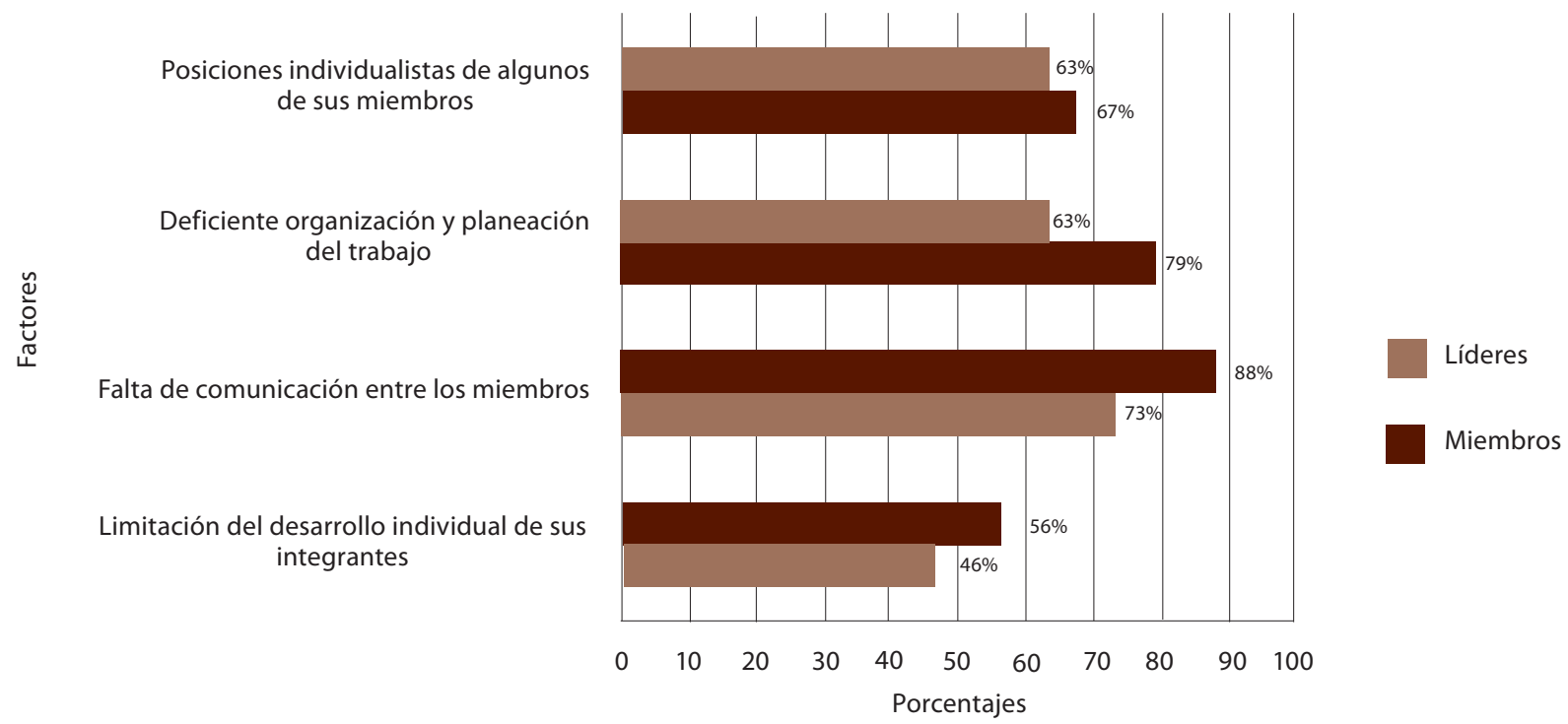

Figura 4. Factores que afectan el buen desempeño de los cuerpos académicos

Al solicitarles a los encuestados algunas recomendaciones para perfeccionar el desempeño de los CA, las más frecuentes estuvieron relacionadas con:

- Fomentar y apoyar la interacción interdisciplinaria entre los cuerpos académicos para llevar a cabo trabajos en conjunto.

- Recibir mayor apoyo institucional y de recursos.

- Se considera que el trabajo de los cuerpos académicos debe de mejorar en dos áreas principales: aumentar su producción científica, mediante un mayor apoyo por parte de las autoridades a los diferentes investigadores, no solo en recursos, sino en promover mas ampliamente la labor de investigación, mediante la descarga académica y administrativa de profesores-investigadores.

- Mayor divulgación de la labor de investigación de los diferentes cuerpos académicos de la facultad y de las restantes de la universidad. 
URL: http://www.una.ac.cr/educare

- Realizar control sistemático de los avances propuestos al interactuar con otras universidades y hacer estancias en las áreas de la especialidad de cada quien, según la institución a la que se desea ir.

- Que los CA sean organismos de evaluación de los alumnos de las carreras con las que se relacionan.

- Que se divulguen los planes de desarrollo y los indicadores de desempeño de los CA.

- Aumentar el reconocimiento e importancia del trabajo en equipo además del individual.

- Realizar mayor número de aplicaciones conjuntas del conocimiento generado.

\section{Conclusiones}

La experiencia adquirida de los cuerpos académicos como un órgano colectivo de trabajo en las universidades debe permitir a cada miembro una visión más completa e integral de las diversas áreas de conocimiento. Sus objetivos y metas abarcan las diferentes áreas de desempeño de los docentes, las cuales, en última instancia, deben influir en un perfeccionamiento constante. La combinación del trabajo colegiado a partir de los aportes individuales de sus miembros permitirá, por una parte, su contribución socio-educativa y, a partir de aquí, la satisfacción personal de los docentes.

El análisis del estado actual de aquellos aspectos más importantes relacionados con el significado y el sentido de los CA en la FIME permite concluir que en esta facultad existen las condiciones objetivas y subjetivas para un perfeccionamiento sistemático de estos importantes órganos. Aunque la mayoría de los líderes y miembros reconocen la importancia del trabajo, se observan una serie de áreas de oportunidades que, una vez superadas, permitirían resultados cualitativamente superiores.

Entre las principales dificultades constatadas se encuentran: limitaciones para el desarrollo del trabajo colaborativo, necesidad de una mayor organización en el trabajo, la falta de concreción y claridad en cuanto a los requisitos de cada una de las etapas de desarrollo de los CA, así como el insuficiente reconocimiento del impacto de estos órganos en los programas educativos y, por ende, en la formación de los estudiantes, así como la necesidad de potenciar en una mayor medida, el vínculo docencia-investigación.

\section{Referencias}

Asociación Nacional de Universidades e Instituciones de Educación Superior (ANUIES). (2000). La educación superior en el siglo XXI. Líneas estratégicas de desarrollo. En ANUIES, Situación, tendencias y escenarios del contexto de la educación superior (Cap. 1, pp. 1-497). Recuperado de http://www.anuies.mx/servicios/d estrategicos/documentos estrategicos/21/sXXI.pdf 
Acosta, A. (2006). Señales cruzadas: Una interpretación sobre las políticas de formación de cuerpos académicos en México. Revista de la Educación Superior, 35(3), 81-92. Recuperado de http://redalyc.uaemex.mx/pdf/604/60413905.pdf

Berman, P. (2000). El estudio de la macro y la micro-implementación. En L. F. Agular (Ed.). La implementación de las políticas (pp. 281-321). México: Porrúa. Recuperado de http://www. inap.org.mx/portal/images/RAP/la\%20implementacion\%20de\%20las\%20politicas.pdf

Facultad de Ingeniería Mecánica y Eléctrica (FIME). (2011). La FIME. Recuperado de http://www. fime.uanl.mx/politicas calidad.php

Fresán, M. (s. f.). Los cuerpos académicos. ¿Por qué cuerpos académicos yno grupos de investigación? Algunas ideas alrededor de los cuerpos académicos (Presentación PowerPoint). Recuperado de http://www.anfei.org.mx/X RGD/merida1.pdf

Grediaga, R. (2000). Profesión académica, disciplinas y organizaciones: Procesos de socialización académica y sus efectos en las actividades y resultados de los académicos mexicanos (Tesis doctoral). México: ANUIES.

Guzmán, T., Hernández, O. y Guzmán, J. (2009). Evaluación e impacto del PROMEP en profesores universitarios. El caso de la Universidad Autónoma de Tamaulipas. Revista Internacional de Ciencias Sociales y Humanidades SOCIOTAM, 19(2), 51-68. Recuperado de http://redalyc. uaemex.mx/src/inicio/ArtPdfRed.jsp?iCve $=65415854003$

López, S. (julio-septiembre, 2010). Cuerpos académicos: Factores de integración y producción de conocimiento. Revista de la Educación Superior, 39(155), 7-25. Recuperado de http:// redalyc.uaemex.mx/src/inicio/ArtPdfRed.jsp?iCve=60418902001

Maldonado-Maldonado, A. (abril-junio, 2005). Comunidades epistémicas: Una propuesta para estudiar el papel de los expertos en la definición de políticas en educación superior en México. Revista de la Educación Superior, 34(2), 134, 107-122. Recuperado de http:// redalyc.uaemex.mx/redalyc/pdf/604/60411920008.pdf

Martínez, P. C., Rico, R. M. y Preciado, S. E. (septiembre-diciembre, 2006). Evolución de los cuerpos académicos en la Universidad de Guanajuato. Acta Universitaria, 16(3),15-24. Recuperado de http://redalyc.uaemex.mx/src/inicio/ArtPdfRed.jsp?iCve=41600302

Olivé, L. (2008). La ciencia y la tecnología en la sociedad del conocimiento. Ética, política y epistemología. México: Fondo de Cultura Económica.

Programa del Mejoramiento del Profesorado (PROMEP). (2006). Reglas de operación e indicadores del programa de mejoramiento del profesorado 2006. México: SEP. Recuperado de http:// promep.sep.gob.mx/reglas/Reglas PROMEP 200627 de Marzo.pdf

Suarez-Núñez, T. y López, L. (enero-abril, 2006). La Organización académica de las universidades públicas: Entre círculos y cuerpos. Contaduría y administración, 218, 147-173. Recuperado de http://contaduriayadministracionunam.mx/articulo-3-104-12.html 
Tierney, W. (2001). La autonomía del conocimiento y el ocaso del estudioso independiente: Posmodernismo y el estudio comparativo de la universidad. Tiempo de educar 3(6), 162188. Recuperado de http://redalyc.uaemex.mx/src/inicio/ArtPdfRed.jsp?iCve=31103607

Universidad Autónoma de Nuevo León (UANL). (2011). Informe de actividades del Sr. Rector Dr. Jesús Ancer Rodríguez correspondiente al 2011. Recuperado de http://www.uanl.mx/sites/ default/files2/informe2011.pdf

\section{Cómo citar este artículo, según APA:}

Dimas, M. I., Torres, A. y Castillo, J. A. (2012). Hacia el perfeccionamiento institucional de los cuerpos académicos en la Facultad de Ingeniería Mecánica y Eléctrica de la Universidad Autónoma de Nuevo León, México. Revista Electrónica Educare, 16(3), 181 202. Consultado de http://www.revistas.una.ac.cr/index.php/EDUCARE/issue/current

Nota: Para citar este artículo en otros sistemas puede consultar el hipervínculo "Como citar el artículo" en la barra derecha de nuestro sitio web:

http://www.revistas.una.ac.cr/index.php/EDUCARE/index 